Mots. Les langages du politique

Le travail et ses maux

\title{
Le néo-management et ses mots : impositions, appropriations, contestations
}

Neo-management and its words: impositions, appropriations, contestations La neogerencia y sus palabras: imposiciones, apropiaciones, contestaciones

Mots. Les langages du politique et Camille Noûs

\section{OpenEdition}

Journals

Édition électronique

URL : https://journals.openedition.org/mots/28228

DOI : $10.4000 /$ mots. 28228

ISSN : $1960-6001$

Éditeur

ENS Éditions

Édition imprimée

Date de publication : 8 juillet 2021

Pagination : $9-13$

ISBN : 979-10-362-0478-4

ISSN : 0243-6450

\section{Référence électronique}

Mots. Les langages du politique et Camille Noûs, « Le néo-management et ses mots : impositions, appropriations, contestations », Mots. Les langages du politique [En ligne], 126 | 2021, mis en ligne le 15 juin 2021, consulté le 17 juin 2021. URL : http://journals.openedition.org/mots/28228 ; DOI : https:// doi.org/10.4000/mots. 28228 


\section{Le néo-management et ses mots : impositions, appropriations, contestations}

\section{Le travail de Mots}

Le projet du présent dossier est né lors du mouvement social de l'automne 2019, qui a débouché sur la constitution du collectif Revues en lutte. Intitulé "Sciences en danger, revues en lutte», l'édito commun que ses membres ont publié au cours de l'année 2020 dénonçait la «crise organisée du service public de la recherche et des universités » menée depuis bientôt quinze ans et consacrée par la récente Loi de programmation de la recherche. Il soulignait les fondements de l' «économie de la connaissance » mise en œuvre par les revues scientifiques et appelait à « défendre l'autonomie de la recherche et de l'édition scientifique» (Collectif des revues en lutte, 2020). Depuis, la gestion de la crise sanitaire a fragilisé encore nos fonctions, que les ministres de l’Éducation nationale et de l'Enseignement supérieur n'ont pas hésité à jeter en pâture à l'extrême droite en lançant une campagne absurde et dangereuse contre les prétendus «islamo-gauchistes » qui «gangrèneraient» l'université (Collectif des revues en lutte, 2021).

L'engagement des revues en lutte se manifeste sous différentes formes, discutées dans les comités de rédaction et débattues au sein du Parlement des revues, fondé en novembre dernier. Mots. Les langages du politique s'est déclarée en lutte en janvier 2020 et a lancé dans la foulée ce numéro spécial sur le travail et ses mots, porté conjointement par la rédaction et Camille Noûs. En anonymisant la codirection du numéro, nous renouons avec une pratique ancienne, que le collectif RogueESR a su réinvestir contre les effets délétères de la bibliométrie sur nos métiers (RogueESR, 2020). Il s'agit pour nous aussi de rappeler la dimension collective de la recherche, qui ne se résume jamais aux quelques chercheuses ou chercheurs que les politiques scientifiques

Mots. Les langages du politique revue.mots@ens-lyon.fr Laboratoire Cogitamus / Cogitamus Laboratory camille.nous@cogitamus.fr 
visent désormais à distinguer et à soutenir au détriment des autres - et de la recherche elle-même. Pour un article publié sous une signature dans notre revue, au moins vingt personnes sont en effet sollicitées, parfois sans autre gratification que celle d'avoir contribué, même modestement, aux progrès de la science : trois évaluateurs, quinze membres du comité éditorial, une secrétaire de rédaction, une maquettiste, des imprimeurs, une ou deux personnes chargées de la communication et de la diffusion, une comptable, etc.

En choisissant de réfléchir collectivement sur la question du travail, nous entendions replacer les attaques contre la recherche et l'enseignement supérieur dans l'entreprise plus large de remise en question du service public et des droits sociaux, pour en éclairer l'un des effets les plus criants, celui qui touche aux conditions de travail et à la façon dont les travailleurs donnent sens à leurs métiers. À cet égard, la conception de ce numéro ne nous a pas conduits à modifier nos habitudes éditoriales. Le choix des objets que nous examinons de dossier en dossier relève évidemment d'un engagement, sinon politique, du moins civique : ils nous semblent soulever des enjeux majeurs pour nos sociétés, que les chercheuses et les chercheurs que nous sommes doivent éclairer et analyser. Mais leur étude doit répondre selon nous à des critères de scientificité exigeants, que nos pairs, sollicités pour expertiser les articles, nous aident à valider et à faire respecter : inscription dans l'état des recherches, mise en œuvre d'une démarche rigoureuse qui explicite ses hypothèses et ses méthodes, objectivation des phénomènes langagiers et sociaux, neutralité axiologique des approches, etc. Contrairement à ce que laissent entendre nos ministres, les sciences humaines et sociales n'ont pas à choisir entre positivisme naîf et idéologie militante: parce que les hommes et les femmes qui s'y engagent font partie de la société qu'ils examinent, ils savent qu'il n'est de point de vue que situé et qu'il leur faut justement l'interroger pour accéder, à travers le débat scientifique, à une description plus adéquate de la réalité.

Celle qui nous intéresse ici concerne les transformations profondes qui affectent le monde du travail depuis les années 1980 au moins et dont les conséquences sont désormais manifestes. Le présent dossiervise ainsi à examiner les discours et les pratiques langagières (Boutet, 2001, 2002) que ces mutations ont suscités et qui les ont aussi, dans une certaine mesure, fait advenir. Comment le travail se dit-il aujourd'hui? Quelles sont les pratiques langagières qui le soutiennent, l'organisent, le structurent? Quelles sont les conceptions politiques sous-jacentes aux manières dont nous en définissons les processus et les pratiques? Et comment, à travers les mots, un ordre politique et social s'impose-t-il ou, au contraire, résiste-t-il ? Telles sont les questions sur lesquelles les auteurs et autrices de ce dossier ont bien voulu se pencher et qu'ils ont accepté de traiter à travers les terrains qui sont les leurs. 


\section{Les avatars du discours néo-managérial}

Dans l'ensemble, le dossier confirme la vitalité du discours néo-managérial, que les échecs économiques et sociaux successifs du néolibéralisme ne semblent pas avoir entamée (Boltanski, Chiapello, 1999; Bezes, 2012 ; Chiapello, Gilbert éd., 2013). À partir d'une enquête menée au sein de trois entreprises françaises, Samir Bedreddine en met au jour l'une des composantes les plus saillantes: le discours de l'innovation porté par des services ou directions dédiés à sa diffusion. Caractérisé par ses emprunts à l'anglais et son imprécision sémantique et actantielle (il faut «penser», «faire », «tester» intransitivement, autrement dit sans objet), ce dernier se révèle à la fois omniprésent et peu efficace. Il a tout en effet de l'injonction contradictoire puisqu'il s'agit d'imposer une nouvelle organisation du travail tout en faisant l'éloge de la liberté au travail. La contradiction place les «managers de l'innovation » dans une position intenable et laisse la majorité des salariés dubitatifs à l'égard d'un discours en grande partie déconnecté de leur expérience de travail.

L'écart est tout aussi grand dans les offres de missions de service civique analysées par Dominique Maillard. Pour leurs rédacteurs, il s'agit d'abord de décrire des activités qui, quoiqu'ancrées dans le monde du travail, ne peuvent l'être dans les termes de la qualification ou de la compétence au risque d'être requalifiées comme des emplois ordinaires. Il leur faut dès lors en passer par la rhétorique de l'engagement, du volontariat et/ou de l'intérêt général, largement empruntée au milieu associatif, qui en dit autant sur les valeurs de leurs promoteurs que sur l'insertion professionnelle des jeunes, dont les enjeux sont partiellement invisibilisés. Comme le discours sur l'innovation, le langage du civisme vise un réenchantement du travail dont on peut aussi se demander s'il passera l'épreuve de la réalité.

Dans son enquête sur les discours prononcés à l'occasion des audiences solennelles de rentrée de la Cour de cassation, Isabelle Huré montre bien que la fonction publique est elle aussi aux prises avec le discours néo-managérial. L'intérêt de son travail est de souligner le travail d'appropriation opéré par les locuteurs - premiers présidents et procureurs généraux - qui s'efforcent d'intégrer les nouvelles normes de la gestion publique à l'histoire longue de leur institution. Ils parviennent ainsi à conforter leurs propres positions, comme garants à la fois des traditions de la Cour et de sa capacité d'adaptation à la modernisation. Le langage de la «modernisation » s'impose ainsi grâce à sa grande malléabilité, qui permet aux cadres chargés de la mettre en œuvre de se l'approprier en l'hybridant avec leurs propres référentiels: celui de la startup dans le cas des «managers de l'innovation », celui du bénévolat dans le cas des offres de missions de service civique, ou celui de la justice dans le cas des hauts magistrats de la Cour de cassation. 


\section{Les langages de la résistance et de la contestation}

Discours et pratiques du néo-management suscitent cependant résistances et contestations, qui apparaissent parfois en filigrane dans les trois premiers textes, mais qui sont au cœur des trois suivants. Pour Agnès VandeveldeRougale, le caractère «hors sol» des stratégies de communication interne des entreprises ne peut déboucher que sur la perte de légitimité des discours d'autorité. L'énoncé «nous ne sommes pas dupes», dont elle étudie le fonctionnement et la circulation, permet ainsi à une multitude d'acteurs de prendre leurs distances à l'égard des promesses de réenchantement du travail que nous évoquions plus haut. La contestation porte là autant contre les artisans du néolibéralisme que contre leurs discours, qui, en travestissant la réalité, minent la confiance indispensable à toute relation de travail et, plus largement, au maintien des institutions.

C'est aussi par le langage - et plus précisément par le témoignage - que des cadres, infirmières et aides-soignantes ayant fait l'expérience du travail en EHPAD cherchent à lutter contre la «maltraitance» des résidents, sans pour autant en rendre leurs anciens collègues responsables. Sophie Béroud et Cristina Nizzoli rendent bien compte de la façon dont elles reprennent le terme de maltraitance au débat public pour en infléchir le sens au profit d'une interprétation systémique des dysfonctionnements du soin en EHPAD: le terme de maltraitance se voit ainsi concurrencé par celui de non-traitance, qui souligne le fait que le problème n'est pas tant l'attitude des soignants à l'égard des résidents que l'impossibilité pour eux de faire correctement leur travail, compte tenu des restrictions budgétaires; la maltraitance devient maltraitance institutionnelle lorsqu'il s'agit d'en imputer la faute aux décideurs, accusés de faire souffrir les résidents autant que les salariés.

Dans l'article qui clôt le dossier, Zoé Haller souligne les contradictions du discours syndical - celui de la Fédération syndicale unitaire (FSU) - lorsqu'il s'agit de lutter pour l'égalité femmes/hommes au travail. Historiquement, les syndicats enseignants ont en effet été à la pointe de ce combat. Leurs cadres semblent néanmoins réticents à s'emparer des mots d'ordre paritaires lorsqu'il s'agit de les appliquer non plus à la fonction publique, mais à l'organisation syndicale elle-même. Les tensions internes qui en résultent sont pour l'autrice symptomatiques des contraintes qui pèsent sur les groupements professionnels (en matière de recrutement notamment) et qui incitent un certain nombre de responsables syndicauxà « évacu[er] les enjeux politiques du paritarisme» (p. 123).

À partir de discours divers, issus de locuteurs aux statuts et emplois très différents, le dossier éclaire ainsi les luttes de représentations qui se jouent au travail et à propos du travail dans le privé comme dans le public, dans le tiers secteur comme dans les organisations professionnelles. 


\section{Références}

Bezes Philippe, 2012, "État, experts et savoirs néo-managériaux: les producteurs et diffuseurs du New Public Management en France depuis les années 1970 », Actes de la recherche en sciences sociales, n0193, p. 16-37.

BoltANSKI Luc, CHIAPELLO Ève, 1999, Le nouvel esprit du capitalisme, Paris, Gallimard.

BOUTET Josiane, 2002, «Pratiques langagières », «Formation langagière », dans P. Charaudeau et D. Maingueneau éd., Dictionnaire d'analyse du discours, Paris, Seuil.

- 2001, «Les mots du travail», dans A. Borzeix et B. Fraenkel éd., Langage et travail: communication, cognition, action, Paris, CNRS Éditions, p.189-202.

ChIAPello Ėve, Gilbert Patrick éd., 2013, Sociologie des outils de gestion: introduction à l'analyse sociale de l'instrumentation de gestion, Paris, La Découverte.

ColleCtIF DES REVUES EN LUTTE, 2021, «Les revues scientifiques exigent la démission de Frédérique Vidal », billet posté sur la plateforme Université ouverte le 16 mars 2021, https://universiteouverte.org/2021/03/16/les-revues-scientifiques-exigent-lademission-de-frederique-vidal/ (consulté le 31 mars 2021).

- 2020, "Sciences en danger, revues en lutte», Mots. Les langages du politique, $\mathrm{n}^{0} 123$, p. 7-15.

RogueESR, 2020, «Alex s'appellera Camille Noûs», billet posté sur la plateforme RogueESR le 16 mars 2020, https://rogueesr.fr/20200316/ (consulté le 31 mars 2021). 